\title{
Linear Dynamics of Ion Sputtered Surfaces: Instability, Stability and Bifurcations
}

\section{Citation}

Davidovitch, Benny, Micahel J. Aziz, and Michael P. Brenner. Forthcoming. Linear dynamics of ion sputtered surfaces: Instability, stability and bifurcations. Journal of Physics: Condensed Matter.

\section{Published Version}

http://www.iop.org/EJ/journal/JPhysCM

\section{Permanent link}

http://nrs.harvard.edu/urn-3:HUL.InstRepos:2795438

\section{Terms of Use}

This article was downloaded from Harvard University's DASH repository, and is made available under the terms and conditions applicable to Open Access Policy Articles, as set forth at http:// nrs.harvard.edu/urn-3:HUL.InstRepos:dash.current.terms-of-use\#OAP

\section{Share Your Story}

The Harvard community has made this article openly available.

Please share how this access benefits you. Submit a story.

\section{Accessibility}




\title{
Linear dynamics of ion sputtered surfaces: Instability, stability and bifurcations
}

\author{
Benny Davidovitch ${ }^{1}$, Michael J. Aziz ${ }^{2}$, and Michael P. Brenner ${ }^{2}$ \\ 1 Physics Department, University of Massachusetts, Amherst, MA 01002 \\ ${ }^{2}$ Harvard School of Engineering and Applied Sciences, Cambridge, MA 02138
}

(Dated: November 11, 2008)

\begin{abstract}
The linear dynamics of ion sputtered solids is essential to understanding the evolution of ordered and disordered surface patterns. We review the existing models of linear dynamics and point out qualitative discrepancies between theory and experimental observations that characterize the linear regime. In particular, we emphasize the importance of experimental and theoretical analysis of bifurcation points: certain values of control parameters such as ion beam angle or energy, where flat surfaces undergo a transition from stability to instability.
\end{abstract}

PACS numbers: 


\section{INTRODUCTION}

The spontaneous evolution of topographic patterns on solid surfaces due to sputtering by a uniform ion beam, a phenomenon which was discovered by Navez et al. in 1962 [1], has attracted high level of interest during the last two decades. As several articles in this volume describe, for a variety of surface types and system parameters (e.g. ion type and energy, beam angle and temperature, substrate type), the evolving surface patterns can take the form of ordered or disordered arrays of one-dimensional ripples or two-dimensional structure of dots, whose typical length scales are $10^{2 \pm 1} \mathrm{~nm}[1-18]$. The large separation of scales between the patterns and the characteristic penetration depth of ions into a solid surface (typically at least an order of magnitude) suggests that evolution of surface morphology can be described as a dynamics of continuous media. In such a formalism [19], pattern formation is attributed to the instability of a homogenously eroding surface to the linear growth of spatially-varying (Fourier) modes, and to the nonlinear interaction between these modes.

The potential use of this method for nanoscale patterning at sub-lithographic length scales has stimulated interest in the control of this phenomenon. Developing such control requires, however, a quantitative nonlinear theory that enables prediction of the type of surface pattern that emerges for a given set of system parameters. Despite significant theoretical effort over the last couple of decades, this goal has not been accomplished yet. Moreover, we will argue below that current experimental data indicate that even for the simplest, prototypical case of isotropic, elemental systems (which lack the potentially confounding effects of crystallographic anisotropy and differential elemental sputtering), we do not yet have a good understanding of even the linear part of the dynamics, which governs the initial formation of the instability. The exploding collection of nonlinear theoretical approaches reduces, in the linear dynamics, to only a handful. We will review here our approach to classifying the possible types of linear dynamics, and will briefly comment on the possible consequences of our studies for the potential development of a nonlinear theory of pattern formation due to ion sputtering.

A widely-used starting point to linear stability analysis was introduced by Bradley and Harper (BH) in 1988 [20]. They built on previous theoretical work of Sigmund [21, 22], who modeled the nuclear energy deposition density (and hence the local erosion rate) as a Gaussian ellipsoid beneath the surface. Sigmund thereby showed that such a response implies 

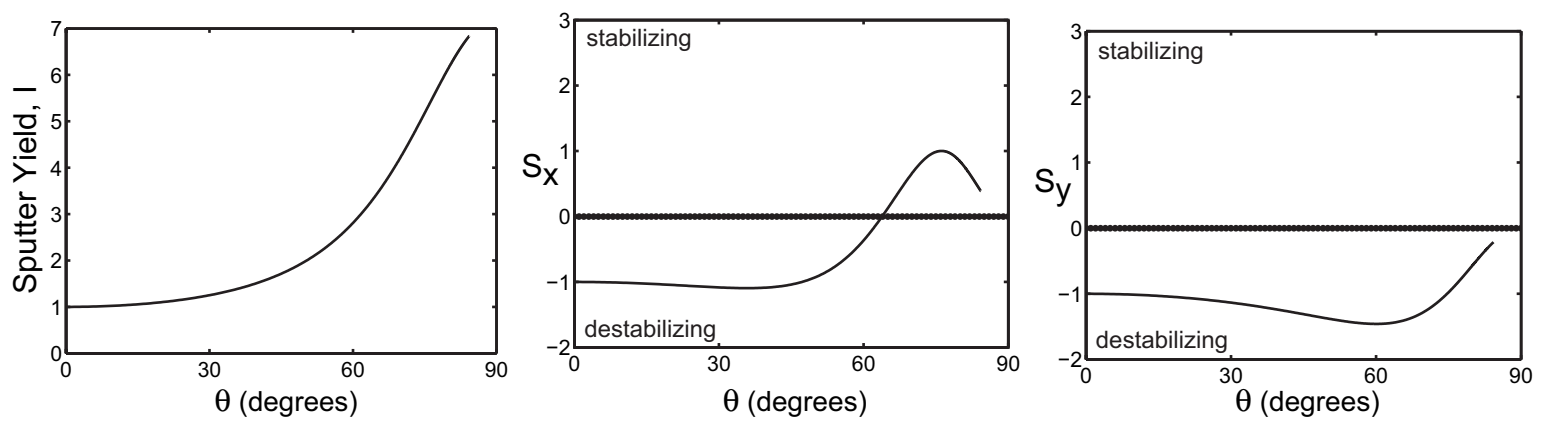

FIG. 1: (a) Plot of sputter yield curve $I(\theta)$, normalized by $I(0)(\mathrm{b}, \mathrm{c})$ Plots of $S_{x}(\theta)$ and $S_{y}(\theta)$, normalized by $\left|S_{x}(0)\right|=\left|S_{y}(0)\right|$. The parameters used are: $a=1.5 \mathrm{~nm}, \sigma=0.9 \mathrm{~nm}, \mu=0.5 \mathrm{~nm}$.

a curvature-dependent sputter yield (atoms out per incident ion), and therefore to a faster erosion of concave surface regions than of convex ones. Assuming an ion beam propagating downward along the $\hat{z}$ direction, Bradley and Harper showed that when Sigmund's response is combined with surface diffusion mediated morphological relaxation [43], the linear dynamics for surface height $h(\mathbf{x}, t)$ of an initially homogenous surface $h=b x$ is:

$$
\frac{\partial h}{\partial t}=-I+\left\{-I^{\prime} \partial_{x}+S_{x} \partial_{x x}+S_{y} \partial_{y y}-\tilde{B} \nabla^{4}\right\} h,
$$

where the coefficients $S_{x, y}(b)$ are derived from Sigmund's Gaussian response function and their negative values reflect the instability mechanism described above. The coefficient $\tilde{B}=\left(1+b^{2}\right)^{-3 / 2} B$, with $B$ is material parameter containing the surface free energy and other material parameters characterizing the kinetics of surface relaxation, and $I(b)$ is Sigmund's sputter yield $\left(I^{\prime} \equiv d I / d b\right)$. For a given beam angle (i.e. given $b$ ) the early evolution of surface morphology is dominated by the fastest growing Fourier modes, and the characteristic pattern length scale is thus $\sqrt{8 \pi^{2} \tilde{B} /\left(-\min \left(S_{y}, S_{x}\right)\right)}$ (assuming at least one of the two BH coefficients $S_{x}, S_{y}$ is negative). We denote by $\theta=\tan ^{-1}(b)$, the angle between the beam direction $(-\hat{z})$ and the normal to the flat surface $\hat{n}(0 \leq \theta \leq \pi / 2)$, and $\hat{x}$ is the axis perpendicular to the beam direction in the $\hat{n}-\hat{z}$ plane [44]. The behavior of $S_{x}(\theta)$ and $S_{y}(\theta)$ for characteristic parameter values is shown in Fig. 1. Bradley-Harper theory gives rise to two central predictions: (i) Below a crossover angle $\theta_{\text {cross }}, S_{x}<S_{y}<0$, implying that for sufficiently small beam angles the emerging pattern is dominated by a parallel mode (i.e. wave vector parallel to projected ion beam direction along the surface) (ii) $S_{y}<0$ for all $\theta$, implying instability of flat surfaces to the growth of perpendicular modes (i.e. wave vector 
perpendicular to ion beam) at all incidence angles. The derivation of nonlinear terms that should be added to the linear BH equation to describe the growth and saturation of pattern amplitude from this model was carried out by Makeev, Cuerno and Barabasi [23, 24], who expanded Sigmund's Gaussian ellipsoid model to higher order in surface height derivatives, and arrived at a Kuramoto-Sivashinsky type equation [19] for the surface evolution.

Although Bradley-Harper theory successfully explains some features of many experiments (e.g. exponential growth and temperature dependence of pattern amplitude [25]) a growing number of experimental observations [14, 26-29] seems to contradict the central predictions of this theory. Some experiments exhibit dominance of perpendicular rather than parallel mode ripples mode at small beam angles, and even more dramatically - beam angle regimes were found where roughness is damped out and flat surfaces are stable and undergo homogenous erosion. These discrepancies are associated with the linear stability of flat surfaces, and thus indicate that $\mathrm{BH}$ linear dynamics are incomplete. (We do not discuss here experiments indicating problems with nonlinear extensions of BH theory [3, 4, 34].) Obviously, linear dynamics is an essential cornerstone for the development of a nonlinear pattern formation theory [19]. These observed discrepancies have thus motivated us to search for a general form of linear dynamics that can capture all existing experimental observations associated with the linear stability and instability and the early stage of pattern growth. The only constraints we suggest to impose on such general linear dynamics are that all its terms must be associated with testable (at least in principle) physical mechanisms, and that its predictions agree with robust features of ion sputtering experiments, most notably the characteristic angular dependence of the sputter yield (see Figs. 1 and 2).

From a mathematical point of view, the possible dynamics that account for the observed deviations from the $\mathrm{BH}$ predictions form two classes [35]: (i) Modifications of coefficients in the $\mathrm{BH}$ equation (1), which allow less restrictive dependence on control parameters (such as beam angle or ion energy), and (ii) Dynamics which require other linear operators to be added to the $\mathrm{BH}$ equation. Physical mechanisms that lead to linear dynamics of class (i) could be associated with local surface responses to ion sputtering which are not of Sigmund's form, or with other local processes such as induced mass flow on the sputtered surface. By contrast, the only examples we have identified that lead to linear dynamics of class (ii) are associated with nonlocal processes, which are described by integral operators whose range is much larger than the pattern wavelength. Moreover, we argue that experimental 
observations support linear dynamics of class (ii). We note that several recent works have suggested various modifications of the $\mathrm{BH}$ equation and its nonlinear extensions [23, 30, 31]. These works were motivated, however, by nonlinear phenomena, most notably by the observation of highly ordered steady patterns $[4,7]$. Although a complete theory for ion-sputtering-induced pattern formation will necessarily include nonlinear components, we believe that an essential prerequisite for such a theory is the understanding of the relevant physical mechanisms in the linear regime. Therefore the analysis and the experimental observations discussed here are associated solely with the behavior in this regime. We point out very briefly how understanding of the linear dynamics may guide our analysis into the nonlinear pattern formation regime.

The paper is organized as follows: In section II we write a very general form for the surface dynamics, and use it to explain the assumptions underlying the BH theory. We review the central predictions of this theory and discuss their robustness with respect to variations in the form of Sigmund response function. Experimental observations that disagree with the $\mathrm{BH}$ predictions are mentioned. In Section III we discuss physically-motivated mechanisms for linear theories of class (i), which preserve the functional form of the BH equation. We show that, although they can potentially explain some of these observations, such theories do not suffice to explain all deviations from the $\mathrm{BH}$ predictions in the linear regime. In Section IV we explain why experimental observations imply terms that must be added to the $\mathrm{BH}$ equations, and point out two examples of these terms containing linear integral operators, hinting on the nonlocal nature of the physical mechanisms that they represent. In Section $\mathrm{V}$ we conclude and discuss directions for future studies inspired by our approach.

\section{BRADLEY-HARPER THEORY AND PURELY EROSIVE RESPONSE}

The most general evolution equation based on the accumulation of local responses to ion impacts is [32]:

$$
\frac{\partial h(\mathbf{x}, t)}{\partial t}=\int \mathbf{d x}^{\prime} J_{i o n}\left(\mathbf{x}^{\prime}\right) \Delta\left[\mathbf{x}-\mathbf{x}^{\prime}, h_{x}(\mathbf{x}, t), h_{y}(\mathbf{x}, t), h_{x x}(\mathbf{x}, t), h_{y y}(\mathbf{x}, t), h_{x y}(\mathbf{x}, t), \ldots\right],
$$

where $\mathbf{x}=(x, y), J_{i o n}\left(\mathbf{x}^{\prime}\right)$ is the ion flux at $\mathbf{x}^{\prime}$, subscripts $x$ and $y$ denote partial derivatives, and the kernel $\Delta\left[\mathbf{x}-\mathbf{x}^{\prime}, \ldots\right]$, representing the change in height at $\mathbf{x}$ due to an ion impact at $\mathbf{x}^{\prime}$, is expected to decay smoothly to zero at large distances $\left|\mathbf{x}-\mathbf{x}^{\prime}\right|$. Assuming radial 
symmetry about the ion track and no explicit dependence on the surface slope and curvature, the simplest form of kernel that describes local response is:

$$
\Delta\left[\mathbf{x}-\mathbf{x}^{\prime}, \ldots\right]=\Delta h(r, z)=-A e^{-g(r)-f(z)}
$$

where $r=\sqrt{\left(x-x^{\prime}\right)^{2}+\left(y-y^{\prime}\right)^{2}}, z=h(x, y)-h\left(x^{\prime}, y^{\prime}\right)$, and $A$ is a length that depends on parameters such as ion energy and ion and target mass. Sigmund's Gaussian ellipsoid response [22] is a particular case of Eq. (3), with

$$
f(z)=\frac{1}{2 \sigma^{2}}(z-a)^{2} \quad ; \quad g(r)=\frac{1}{2 \mu^{2}} r^{2}
$$

where $a$ is the average penetration depth of the ion, and $\sigma, \mu$ are lengths characterizing the ranges of response in directions parallel and perpendicular to $\hat{z}$, respectively.

Bradley and Harper [20] assumed Sigmund's response (4) and carried out an expansion of $h(x, y, t)$ around the homogenously flat surface $h=b x-I t$ (where $I$ is the average vertical erosion rate). Let us notice already that a similar expansion can be pursued whenever a kernel $\Delta$ in Eq. (2) is assumed to be characterized by scales (e.g. $a, \mu, \sigma$ in Eq. (4)) much smaller than the characteristic scales of the evolving surface patterns. The BH analysis yields Eq. (1) with the terms $S_{x} h_{x x}$ and $S_{y} h_{y y}$ on its RHS. The small parameter of such an expansion is proportional to the ratio between a characteristic length of Sigmund's response (e.g. penetration depth $a$ ) and a typical wavelength of the evolving pattern. This implies two important consequences: First - the approach is valid only if the unstable wavelength is indeed sufficiently larger than $a$ (or some other length characterizing the local response). Second - linear terms in this expansion that are proportional to higher order derivatives of $h$ can be neglected (with respect to $S_{x} h_{x x}$ and $S_{y} h_{y y}$ ). In terms of Sigmund's parameters, the sputter yield and $\mathrm{BH}$ coefficients are given by the formulas:

$$
\begin{aligned}
I(b) & =\alpha \int_{-\infty}^{\infty} d y \int_{-\infty}^{\infty} d x e^{-\rho_{b}(x, y)} \\
S_{y}(b) & =\alpha \int_{-\infty}^{\infty} d y \int_{-\infty}^{\infty} d x e^{-\rho_{b}(x, y)} f^{\prime}(b x) y^{2} \\
S_{x}(b) & =\alpha \int_{-\infty}^{\infty} d y \int_{-\infty}^{\infty} d x e^{-\rho_{b}(x, y)} f^{\prime}(b x) x^{2}
\end{aligned}
$$

where $\rho_{b}(x, y)=g\left(\sqrt{x^{2}+y^{2}}\right)+f(b x)$ and $\alpha$ is a constant. Characteristic plots of these coefficients (as a function of slope $b$ ) are given in Fig. 1. Analysis of these expressions yields two central predictions: (i) The $\mathrm{BH}$ coefficient $S_{y}$, associated with the dynamics of Fourier 
modes $h(x, y, t)=b x-I t+e^{R_{q} t} \cos (q y)$ is negative for all values of $b$, implying $R_{q}>0$ and hence instability of the sputtered surface to growth of perpendicular mode ripples. This consequence applies for all values of Sigmund's parameters $a, \sigma, \mu$. (ii) Assuming the Gaussian ellipsoid response shape, with $\sigma>\mu$ (corresponding to a broader distribution of the nuclear energy deposition along along its trajectory direction than in the perpendicular directions, on average), one finds $S_{x}<S_{y}<0$ for sufficiently small beam angles (i.e. small slope $b$ ). The wavelength of the unstable modes (associated with negativity of $S_{y}$ and of $S_{x}$ for sufficiently small $b$ ) is determined by a relaxation mechanism. BH assumed a suggested a Mullins-Herring surface diffusion mediated relaxation [33], as represented by the term $-\tilde{B} \nabla^{4} h$ on the RHS of Eq. (1). With typical values of $\tilde{B}$ and Sigmund's parameters of order $\sim 1 \mathrm{~nm}$ (for typical ion energies of order $1 \mathrm{keV}$ ), one finds characteristic dominant (fastest growing) pattern wavelength $\sqrt{8 \pi^{2} \tilde{B} /\left(-\min \left(S_{y}, S_{x}\right)\right)}$ of order tens to hundreds of nm's, in agreement with experimental observations. As discussed in the introduction, these predictions have been contradicted by some recent experiments.

The above review of the $\mathrm{BH}$ approach suggests a natural anchor for modifications of the linear theory: the response function $\Delta$ in Eq. (2). Although Sigmund's Gaussian ellipsoidal response is plausible and has been widely accepted, it is important to remember that the "microscopic" coefficients $a, \sigma, \mu$, and moreover the Gaussian ellipsoidal shape of the surface response, are not measured directly in experiments. The strongest experimental evidence in favor of Sigmund's response is the angular dependence of the sputter yield $I(b)$, whose qualitative shape (up to large slopes $b>1$ ) as plotted in Fig. $1\left(I(0)>0, I^{\prime}(0)=0\right.$, and monotonic increase with $b>0$ ) have been verified in many experiments. In contrast, the BH coefficients $S_{x}, S_{y}$ are not measured directly in most experiments.

Assuming another, non-Sigmund type of local response $\Delta$, the $\mathrm{BH}$ expansion as described above can be repeated, and modified coefficients $S_{x, y}^{e f f}$ can be computed. As long as the assumed response $\Delta$ is local, namely its characteristic scales are much smaller than the evolving pattern wavelength, such a modification preserves the linear functional form of the $\mathrm{BH}$ equation (1). Our first question is thus: Which of the BH predictions are robust? i.e., which predictions remain valid under a modification of the local kernel $\Delta$ that does not significantly affect the sputter yield $I(b)$ ?

Results from our investigation of this problem [35] are presented in the rest of this section and in the next one. Here we discuss a natural generalization of Sigmund's response to 
functions of the form (3) where the functions $f(z)$ and $g(r)$ are not necessarily quadratic, as assumed in Sigmund's form (4). Numerical evidence for the plausibility of such generalized form comes from the work of Feix el at. [30], who used Molecular Dynamics methods to simulate the energy deposited by impinging ion. Their numerical simulations yielded a "toroidal" response function of the form (3) with $g(r)+f(z)$ attaining its minimal value (thereby maximizing the deposited energy density) along a ring $\left(z=0, r=r_{0}>0\right)$ rather than at the "average ion stopping point" $(z=0, r=0)$. Motivated by this result, we repeated the $\mathrm{BH}$ expansion, using for $\Delta$ the form (3) with non-quadratic functions $g(r)$ and $f(z)$ [35]. We thus arrived at modified BH coefficients $S_{x}^{e f f}, S_{y}^{e f f}$, such that the integral on the RHS of Eq. (2) is approximated by the differential operators: $S_{x}^{e f f} h_{x x}+S_{y}^{e f f} h_{y y}$. We studied general smooth functions $f(z)+g(r)$ that assume minima at a single point $(z=0, r=0)$, and along a curve $(z=0, r>0)$. In order to compute the modified BH coefficients $S_{x, y}^{e f f}$ we used the saddle point technique. Our detailed calculations are presented in section III of [35]. A central result of our analysis is that all such response functions give rise to $S_{y}^{e f f}(b)<0$, and therefore to instability of flat surfaces with respect to amplification of perpendicular modes, for all beam angles. Furthermore, although the calculations become increasingly cumbersome as more complicated forms of the functions $g(r), f(z)$ are considered, our result suggests that $S_{y}^{e f f}(b)<0$ for all beam angles, as long as the surface response to local ion $\Delta$ is purely erosive, namely - gives rise to net erosion at all surface points. Such a response can be represented by a function of the type (3) or by the more general form:

$$
\Delta h(r, z)=-\sum_{j=1}^{\infty} A_{j} e^{-g_{j}(r)-f_{j}(z)},
$$

with coefficients $A_{j}>0$ [45]. We may thus conclude that the $\mathrm{BH}$ prediction of the instability of a flat surface at any beam angle is fairly robust with respect to modifications of the response function to ion impact that are erosive everywhere on the surface. Motivated by this conclusion, we went on to explore the robustness of the instability of flat surfaces when local responses which are not everywhere erosive are considered. This issue is discussed in the next section.

Another important conclusion is that the $\mathrm{BH}$ prediction of the dominance of parallel modes over perpendicular modes at small beam angles does not seem to be as robust as the instability prediction. This can be easily demonstrated by considering e.g. a Gaussian ellipsoid with $\mu>\sigma$. Such a response is obviously erosive everywhere, yet it gives rise to 
dominance of perpendicular modes at small beam angles.

\section{MODIFICATIONS OF BH COEFFICIENTS}

The conclusions of our work discussed in the previous section is that a purely erosive, local surface response to ion impact of the form (2) is contradicted by experimental observations of a stable flat surface over a range of incident angles. In this section we discuss surface dynamics of the general type (2), where the assumption that the response is of the purely erosive type (3) or (6) with $\left\{A_{j}>0\right\}$ is relaxed. Our discussion is limited to two representative response types, whose corresponding sputter yield is similar to the one obtained for Sigmund's response, but which may give rise to beam angles for which flat surfaces are stable.

\section{A. Response that is not erosive everywhere}

Several recent studies demonstrated that under certain circumstances it is possible that a crater forms at the impact point and is surrounded by a rim elevated from the original surface $[5,8,36,37]$. This behavior, where $\Delta h>0$ in the rim, is qualitatively different from the erosive response functions described in (3). We then ask: Is the BH prediction of the instability of a flat surface at all beam angles robust when such a response is considered?

To carry out this analysis we considered response functions of the form (6) where some of the coefficients $A_{j}$ are negative and some are positive. Whereas arbitrary choices of the coefficients $A_{j}$ give rise to a yield curve $I(b)$ substantially different from the shape depicted in Fig. 1 (and even to overall deposition rather than erosion of the surface), it is possible to show that there exist choices of coefficients $\left\{A_{j}\right\}$, which do preserve the yield curve closely. It is this class of coefficients we are interested in. Focusing for simplicity on a response function that can be approximated as the sum of two Gaussian ellipsoids with positive and negative coefficients, respectively:

$$
\Delta h(r, z)=-A\left[e^{-r^{2} / 2 \mu_{1}^{2}-\left(z-a_{1}\right)^{2} / 2 \sigma_{1}^{2}}-\beta e^{-r^{2} / 2 \mu_{2}^{2}-\left(z-a_{2}\right)^{2} / 2 \sigma_{2}^{2}}\right],
$$

where $A, \beta>0$, we shoed [35] that if $\beta<\beta^{*}\left(\mu_{1,2}, \sigma_{1,2}\right)$ the yield curve is not significantly modified from the form predicted by Sigmund's response, to within experimental error, 
where:

$$
\beta^{*}=\left(\mu_{1} / \mu_{2}\right)^{2} e^{a_{2}^{2} /\left(2 \sigma_{2}^{2}\right)-a_{1}^{2} /\left(2 \sigma_{1}^{2}\right)}
$$

Moreover, we showed that although the condition (8) generally implies a small ratio between the rim and crater amplitude, it is possible to find parameter regimes where $S_{y}^{e f f}(b)>0$ (i.e. stability to perpendicular modes) at some intervals of the slope $b$, unlike the case for response functions discussed in the previous section [35]. In particular, one can find parameter regimes where $\beta<\beta^{*}$ (thus the yield curve retains its characteristic shape) and $S_{x}^{e f f}(b), S_{y}^{e f f}(b)$ are both positive over various intervals of the slope $b$. A yield curve $I(b)$, and modified BH coefficients $S_{x}^{e f f}(b), S_{y}^{e f f}(b)$ for two response functions of the type (7) with representative sets of parameters $\mu_{1,2}, \sigma_{1,2}, \sigma$ are plotted in Fig. 2. While both give rise to essentially identical $I(b)$ (whose variation from the shape predicted by Sigmund's response, obtained by $\beta=0$, is unnoticeable), the modified $\mathrm{BH}$ coefficients $S_{x}^{\text {eff }}(b), S_{y}^{\text {eff }}(b)$ are markedly different from the shape predicted by Sigmund. Notably, regimes of beam angle (i.e. surface slope) where flat surface is stable (i.e. both $S_{x}^{e f f}(b)>0$ and $S_{y}^{e f f}(b)>0$ ) can be obtained for small beam angles or even for intermediate values of beam angles, in qualitative agreement with the experiments of [14] and [27]. This result demonstrates a very significant conclusion: small changes in the shape of the surface response of a single ion can completely change the stability characteristics of a flat surface from BH predictions, but yet lead to an experimentally indistinguishable sputter yield curve. Further analysis will require a microscopic theory for sputtering processes which are not erosive everywhere. Alternatively, comparison with atomistic simulations [37] may allow extraction of effective parameters such as $\beta, a_{1,2}, \sigma_{1,2}$, and $\mu_{1,2}$ in Eq. (7).

\section{B. Induced surface currents}

Another possible type of local response that qualitatively modifies the $\mathrm{BH}$ coefficients but not the functional form of the BH equation (1) is related to surface currents induced by ion impact. The existence of such surface currents was conjectured by Carter and Vishnyakov [26], who associated them with an average forward motion of recoils parallel to the ion direction before coming to rest. Recently, this effect was observed by Moseler et al. [38] in MD simulations in the study of the ion-enhanced smoothing of diamond-like carbon surfaces bombarded by low energy $(30-150 \mathrm{eV})$ carbon ions at near-normal incidence. These 

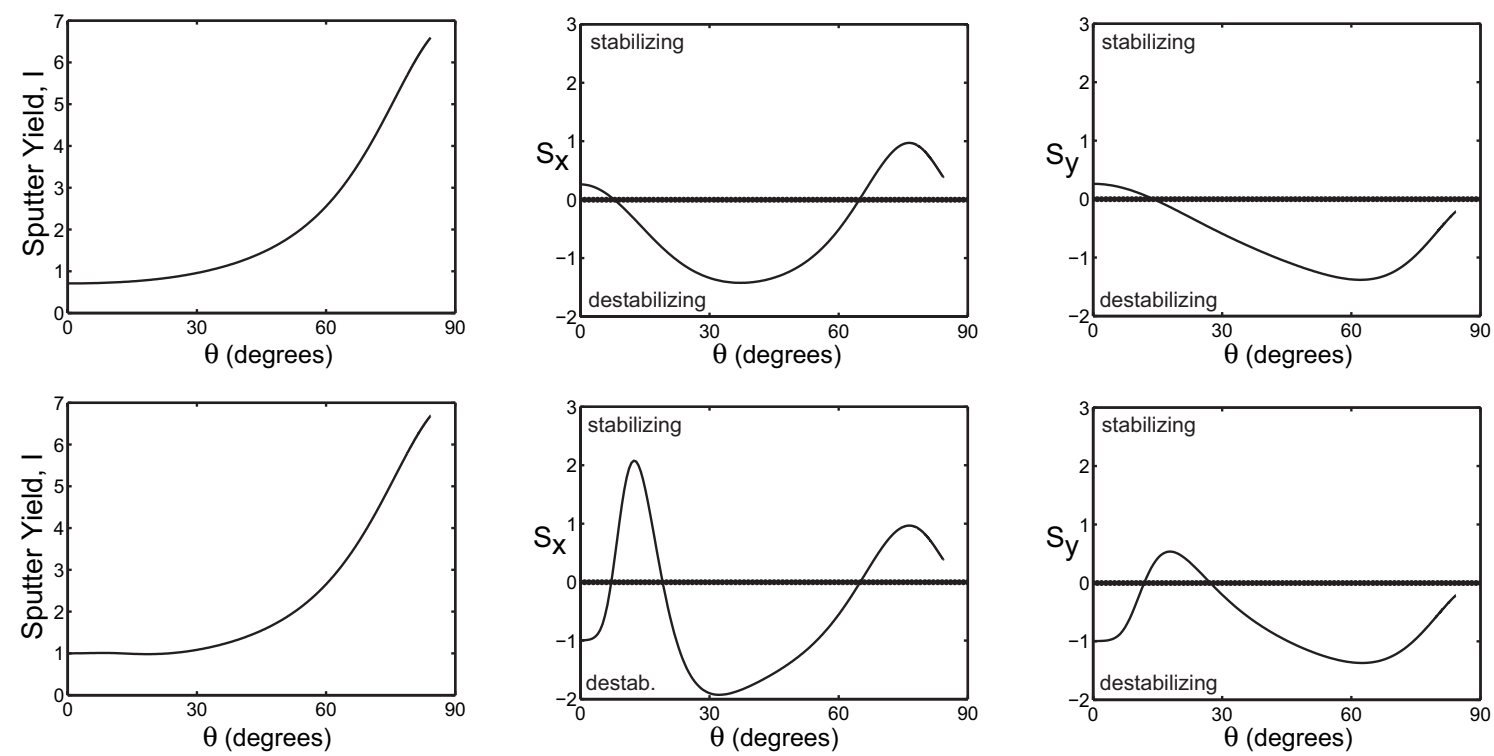

FIG. 2: Normalized yield curve and BH coefficients $S_{x}, S_{y}$ for two sets of parameters of the twoGaussians model, Eq. (7). The parameters $a_{1}, \sigma_{1}, \mu_{1}$ are the "Sigmund parameters" taken as in Fig. 1, and the same normalization factors are used. The new parameters are: top row $\alpha=0.03 ; a_{2}=0.5 \mathrm{~nm}, \sigma_{2}=0.5 \mathrm{~nm}$, and $\mu_{2}=1 \mathrm{~nm}$; bottom row $-\alpha=0.03, a_{2}=0.9 \mathrm{~nm}, \sigma_{2}=0.2$ $\mathrm{nm}$ and $\mu_{2}=1.5 \mathrm{~nm}$.

simulations found that the average net effect of each ion impact is a displacement along the surface that is proportional to $\theta$ for small beam angle $\theta$. Induced surface currents also appear to be important for the stability of crystalline surfaces under growth and erosion [39]. The mechanisms of [26] and [38] are somewhat different: the former, developed to model the response to high-energy ions, assumes volume transport in the collision cascade with a component parallel to the surface that yields a net current, whereas the latter seems to be a low-energy effect where a true surface current is induced. However, in both cases an explicit dependence on angle of incidence is apparent, and phenomenologically they appear virtually indistinguishable.

Such induced surface currents can be modeled using a local response function (3), but with an important difference from the analysis described so far in the above sections. The response functions (3) or (6) do not depend explicitly on the incidence angle and are fully characterized by considering normal incidence $(b=0)$. For such functions the dependence of the coefficients $I(b), S_{x}(b)$ and $S_{y}(b)$ in Eq. (1) on the angle $\theta=\tan ^{-1}(b)$ is purely 
geometrical, reflecting the fact that the distribution of values of the ion trajectory projections on the surface depends on the slope $b$. In contrast, local responses $\Delta$ that reflect also induced surface currents are expected to depend explicitly on slope, and cannot be written in the $b$ independent forms (3) or (6). Incorporating induced surface currents into surface dynamics yields on the RHS of Eq. (1) the linear terms $\nu_{x} h_{x x}+\nu_{y} h_{y y}$, where

$$
\begin{aligned}
& \nu_{x}(b) \propto \frac{1-b^{2}}{\left(1+b^{2}\right)^{2}} \\
& \nu_{y}(b) \propto \frac{1}{1+b^{2}},
\end{aligned}
$$

and the proportionality coefficients are positive constants that can depend on surface type, temperature, ion type and energy [26, 35].

Let us highlight two important points regarding this analysis. First, surface currents conserve mass and thus do not affect the sputter yield $I(b)$. Therefore, it is impossible to confirm or rule out their existence from sputter yield measurements. Second, although surface currents cannot be represented by local response of the form (3) or (6), their effect on the linear surface dynamics is similar and is captured through terms proportional to secondorder partial derivatives. Incorporation of induced surface currents into ion-sputtered surface dynamics thus preserves the functional form of Eq. (1), and amounts to adding to the BH coefficients $S_{x}^{e f f}, S_{y}^{\text {eff }}$ terms of the same order with coefficients $\nu_{x}(b), \nu_{y}(b)$, respectively. The possible effect of surface currents on the stability of flat surfaces is qualitatively similar to

the schematic plot in the top row of Fig. 2. If the induced surface current mechanism is sufficiently strong, one should observe a regime of stable flat surfaces at small beam angles. Although this mechanism could be related to the observation of flat surfaces in experiments [26], stability of flat surfaces at a range of intermediate beam angles, reported in [14] and [27] seems to imply other mechanisms not included in this approach, such as response functions that are not everywhere erosive (discussed in the beginning of this section) and possibly other processes as described in the following sections.

\section{FINITE WAVELENGTH AND NONLOCAL PROCESSES}

There are two common features to the mechanisms described in the previous two sections. First, they are all associated with process whose effect on the surface dynamics can be described by a local response $\Delta$ in Eq. (2), which depends only on the local topography of 


$$
\begin{array}{lll}
\text { (a) } R_{q}=-S^{\text {eff }} q^{2}-B q^{4} & \text { (b) } R_{q}=-K-S^{\text {eff }} q^{2}-B q^{4} & \text { (c) } R_{q}=-S^{\text {eff }} q^{2}+M\left|q^{3}\right|-B q^{4}
\end{array}
$$
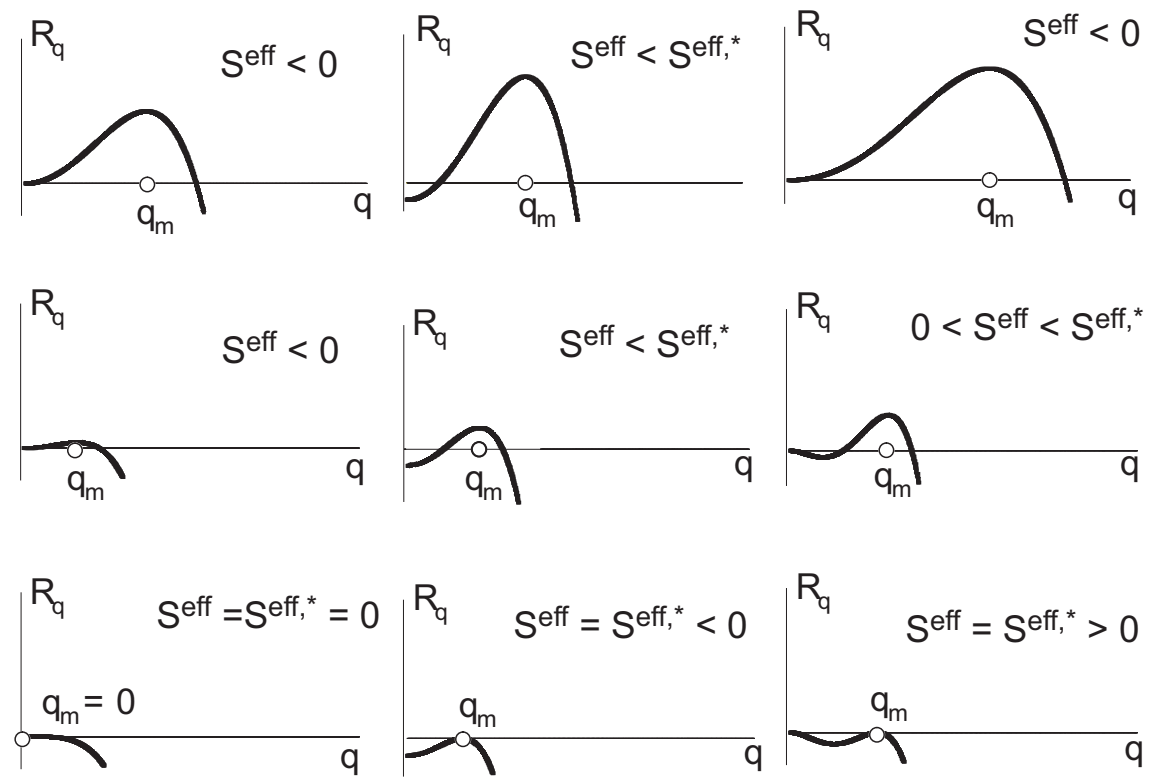

FIG. 3: Schematic plots depicting the transition between stable and unstable surface dynamics for three dispersion relations. (a) Left column: generalized Bradley-Harper, Eq. (1), where the transition occurs at $S^{\text {eff,* }}=0$ with diverging wavelength. (b) Middle column: with Facsko nonlocal "damping term", transition occurs at $S^{\text {eff, } *}<0$ with finite wavelength. (c) Right column: with Asaro-Tiller nonlocal elastic energy mechanism, transition occurs at $S^{\text {eff,* }}>0$ with finite wavelength.

the surface point that undergoes ion impact. Second, they all give rise to surface dynamics of the form (1), whose possible bifurcations - transitions between stability and instability of flat surface - are associated with vanishing amplitude and divergence of the pattern wavelength at certain beam angles. This is depicted in the left column of Fig. 3, where we schematically plot the growth rate $R_{q}$ of Fourier modes with wavenumber $q$ for values of $b$ above bifurcation (where there exist unstable modes with $R_{q}>0$ ), at bifurcation, and below bifurcation (where all modes are decaying $R_{q}<0$ ). Mathematically, this is reflected through the fact that bifurcation corresponds to parameter values where the maximal growth rate (over all Fourier modes) is vanishing: $\max \left(R_{q}\right)=0$. Because the dispersion relation from (1) but with $S$ replaced by $S_{\text {eff }}$ is $R_{q}=S_{j}^{e f f} q^{2}-B q^{4}$ (where $i=x, y$, for parallel or perpendicular modes, respectively, and where $S_{\text {eff }}$ corresponds to any modifications of 
the $\mathrm{BH}$ coefficients associated with local response as described in previous section), we see that bifurcation described by surface dynamics (1) implies that the typical length scale (proportional to the inverse wavenumber of the most unstable mode), must diverge.

While stability of flat surfaces at various regimes of beam angles has been noticed in the past [26], there exists only a handful of reports in the literature on wavelength measurements near bifurcation. While it is hard to draw general conclusions from few existing results, there is a substantial evidence that at least some bifurcation points in the dynamics of ion-sputtered surfaces are characterized by finite rather than diverging wavelength [27-29]. This observation, together with the above argument, suggest that linear surface dynamics is not fully described by an equation of the form (2), but is rather influenced by nonlocal processes, whose characteristic spatial range is not necessarily smaller than the scale of the emerging pattern. General conditions under which this assertion applies are listed in [35]. Here we will demonstrate this principle through two examples.

\section{A. Facsko "damping" term}

The schematic plots in the middle and right columns of Fig. 3 demonstrate the qualitative effect of such additional mechanisms on surface dynamics near bifurcation. The middle column describes a bifurcation associated with variation of a modified $\mathrm{BH}$ coefficient $S^{e f f}=$ $S_{x}^{e f f}$, through the variation of a control parameter (e.g. beam angle or ion energy), where the growth rate $R_{q}$ is assumed to be dominated by:

$$
R_{q}=-K+S^{e f f} q^{2}-\tilde{B} q^{4}
$$

and for simplicity we assume the unstable mode is along the $x$-direction. The center column of Fig. 3 reflects the fact that the equation $\max \left\{R_{q}\right\}=0$, which represents the bifurcation point, selects a most unstable wavenumber $|q|>0$ as bifurcation is approached. To understand why Eq. (11) reflects a nonlocal process, notice that the constant $-K$ on its RHS correspond to a "damping" linear term $\partial h / \partial t=-K h(\mathbf{x}, t)$ in the real-space surface dynamics (in addition to any other terms associated with local response in Eq. (2)). However, because the dynamics must be invariant under translation $h \rightarrow h+c$ of the surface level (where $c$ is any constant), such a term must appear as a part of an integral term $-K\left[h(\mathbf{x}, t)-\int d \mathbf{x}^{\prime} h\left(\mathbf{x}^{\prime}, t\right)\right]$ that preserves this invariance. Such a term was proposed by 
Facsko et al. [31] in order to explain the observations of ordered arrays of ripples and dots in experiments and simulations [4, 7, 14]. Its physical origin, hypothesized to be related to nonlocal redeposition, has not been clarified yet. The emergence of a spatial integral over the whole sputtered surface associated with this term is an example of the principle mentioned above: Finite wavelength near bifurcation results from a surface response over scales much larger than the nanometric scales associated with penetration depth and energy release of the ion.

\section{B. Asaro-Tiller stresses}

Another example that yields a bifurcation with finite wavelength upon variation of a modified BH coefficient $S^{\text {eff }}$, is depicted in the right column of Fig. 3:

$$
R_{q}=S^{e f f} q^{2}+M\left|q^{3}\right|-\tilde{B} q^{4}
$$

where again we assume for simplicity that the dominant wave vector near bifurcation is along the $x$-direction, and denote by $S^{e f f}=S_{x}^{e f f}$, the modified BH coefficient. As in the previous example, bifurcation is represented by $\max \left\{R_{q}\right\}=0$, which is obtained for $q=M / 2 B>0$.

The growth rate $R_{q}$ in Eq. (12) stems from adding a term $M|q|^{3}$ associated with induced stresses in the solid to the "usual" terms $S^{e f f} q^{2}$ and $-\tilde{B} q^{4}$ associated with local response in Eq. (1). This term is related to the well-known Asaro-Tiller instability, which develops on surfaces of solids subject to biaxial stress $\sigma_{0}[40,41]$. The coefficient $M \propto \sigma_{0}^{2}$, where $\sigma_{0}$ is the induced stress in the near surface region due to the ion bombardment. The nonlocal mechanism associated with the Asaro-Tiller term is less transparent than the nonlocality associated with the damping term $-K$ in the growth rate (11), and its derivation is based on elasticity theory, which we will not discuss here. It suffices to mention that the term $M|q|^{3}$ in $R_{q}$ corresponds to a real-space surface dynamics $\partial h / \partial t$ arising from a long-range elastic interaction and dominated by a (spatial) integral over the whole surface, similar to the integral associated with the damping term $-K$. From a mathematical perspective, the dependence of $R_{q}$ on a nonanalytic function of $q$ (here $|q|^{3}$ ) indicates that this term is not related to a local (i.e. differential) operator such as $\nabla^{3}$, but rather to some linear integral of $h(\mathbf{x}, t)$.

Although the physical mechanism underlying the growth rate (12) is much clearer than the 
global redeposition process that may give rise to a growth rate of the type (11), preliminary measurements of the induced stress $\sigma_{0}$ (hence the coefficient $M$ in (12)) indicate that $M$ is too small to affect a qualitative change in the linear dynamics from the shape in Fig. 3a to the shape in Fig. 3b [42]. Thus, the actual mechanism underlying nonlocal response which eventually leads to finite pattern wavelength at bifurcation remains unclear.

\section{CONCLUSIONS AND OUTLOOK}

We described here a theoretical approach for discerning the general form of the linear dynamics of ion sputtered surfaces by focusing on the analysis near bifurcation points, where a flat surface becomes unstable to the formation of a topographic pattern upon the smooth variation of a control parameter (e.g. beam angle or energy). Our analysis, together with recent experimental observations, suggests that the dynamics are strongly affected by physical processes that cannot be described as a local response of the surface to ion impact. This conclusion is arrived at by contrasting our theoretical observation that local response implies diverging pattern wavelength at putative bifurcations with experimental observations that clearly indicate the existence of a bifurcation without a diverging length scale.

The actual mechanisms required to explain existing data, however, are still unclear. We showed that several mechanisms beyond the classical theory of Bradley and Harper can be relevant in developing a linear dynamics theory that explains all existing data, but it is quite possible that some of these mechanisms act simultaneously. For example, observations of a stable flat surface over various regimes of beam angle can be related to local response (2) to ion impact which are not purely erosive, as described in section III, but observations of finite pattern wavelength near bifurcations seem to reflect the influence of nonlocal mechanisms on the surface dynamics. At the current stage, it is hard to conclude which of the mechanisms outlined in the sections III and IV are actually relevant, because existing models and experimental measurements of relevant physical parameters are not sufficiently quantitative. We believe that a prime goal of research in this field is the development of the general linear dynamics underlying the early stage of pattern formation. As we described here, the identification of bifurcation points and a careful analysis of pattern features in their vicinity is an extremely valuable tool for that purpose.

Finally, we mention that although the focus of this review was on linear dynamics, we 
expect the outcome of this study to be an essential cornerstone for the development of a nonlinear pattern formation theory, which will eventually allow the prediction of pattern features for a given set of control parameters. This expectation is based on the universal properties of weakly nonlinear pattern formation theory: quite generally [19], knowledge of the linear dynamics and the associated leading order nonlinearity (often extracted from symmetry principles) suffices to classify the possible pattern morphologies near and further away from bifurcation points.

\section{Acknowledgments}

The research of MJA was supported through DE-FG02-06ER46335, the research of BD was supported through the Harvard MRSEC, and the research of MPB was supported through NSF-0605031. We thank W. Van Saarloos for bringing to our attention the importance of a careful analysis of the stable-unstable transition. We thank Bashkim Ziberi, Charbel S. Madi, H. Bola George, and Scott Norris for many helpful discussions.

[1] M. Navez, D. Chaperot, and C. Sella, C. R. Hebd. Seances Acad. Sci. 254, 240 (1962).

[2] R. L. Meek, W. Gibson, and J. Sellscho, Appl. Phys. Lett. 18, 535 (1971).

[3] J. Erlebacher, M.J. Aziz, E. Chason, M. Sinclair, and J. Floro, Phys. Rev. Lett. 82, 2330 (1999).

[4] S. Facsko et al., Science 285, 1551 (1999).

[5] G. Costantini, E. De Mongeot, C. Boragno, and U. Valbusa, Phys. Rev. Lett. 86, 838 (2001).

[6] S. Habenicht, Phys. Rev. B 63 (2001).

[7] S. Facsko, T. Bobek, T. Dekorsy, and H. Kurz, Phys. Stat. Solidi B 224, 537 (2001).

[8] I. Yamada, J. Matsuo, N. Toyoda, and A. Kirkpatrick, Mat. Sci. Eng. R 34, 231 (2001).

[9] C. Umbach, R. Headrick, and K. Chang, Phys. Rev. Lett. 8724 (2001).

[10] U. Valbusa, C. Boragno, and F. De Mongeot, J. Phys.: Condens. Matter 14, 8153 (2002).

[11] E. Chason and M.J. Aziz, Scr. Mater. 49, 953 (2003).

[12] A. Brown and J. Erlebacher, Phys. Rev. B 72 (2005).

[13] A. Cuenat, H. George, K. Chang, J. Blakely, and M.J. Aziz, Adv. Mater. 17, 2845 (2005). 
[14] B. Ziberi, F. Frost, T. Hoche, and B. Rauschenbach, Phys. Rev. B 72 (2005).

[15] B. Ziberi, F. Frost, and B. Rauschenbach, J. Vac. Sci. Technol. A 24, 1344 (2006).

[16] Y. R. Kim, P. Chen, M. J. Aziz, D. Branton, and J. J. Vlassak, J. Appl. Phys. 100 (2006).

[17] W. L. Chan and E. Chason, J. Appl. Phys. 101 (2007).

[18] R. Gago et al., Nanotechnology 13, 304 (2002).

[19] M. Cross and P. Hohenberg, Rev. Mod. Phys. 65, 851 (1993).

[20] R. M. Bradley and J. M. E Harper, J. Vac. Sci. Technol. A 6, 2390 (1988).

[21] P. Sigmund, Phys. Rev. 184, 383 (1969).

[22] P. Sigmund, J. Mat. Sci. 8, 1545 (1973).

[23] M. Makeev, R. Cuerno, and A. Barabasi, Nucl. Sci. Eng. 197, 185 (2002).

[24] R. Cuerno and A. Barabasi, Phys. Rev. Lett. 74, 4746 (1995).

[25] J. Erlebacher, M.J. Aziz, E. Chason, M. Sinclair, and J. Floro, J. Vac. Sci. Technol. A 18, 115 (2000).

[26] G. Carter and V. Vishnyakov, Phys. Rev. B 54, 17647 (1996).

[27] C. S. Madi, B. Davidovitch, H. B George, S. A. Norris, M. P. Brenner, and M. J. Aziz, Phys. Rev. Lett. (in press).

[28] H. Zhou et al., Phys. Rev. B. 75, 155416 (2007).

[29] B. Ziberi, Ph.D. thesis, Leipzig University, 2005.

[30] M. Feix, A. Hartmann, R. Kree, J. Munoz-Garcia, and R. Cuerno, Phys. Rev. B 71 (2005).

[31] S. Facsko, T. Bobek, A. Stahl, H. Kurz, and T. Dekorsy, Phys. Rev. B 69 (2004).

[32] M.J. Aziz, Mat. Fys. Medd. K. Dan. Videnk. Selsk. 52, 187 (2006).

[33] W. Mullins, J. Appl. Phys. 30, 77 (1959).

[34] A. Brown, J. Erlebacher, W. Chan, and E. Chason, Phys. Rev. Lett. 95 (2005).

[35] B. Davidovitch, M. J. Aziz, and M. P. Brenner, Phys. Rev. B 76 (2007).

[36] E. Bringa, K. Nordlund, and J. Keinonen, Phys. Rev. B 64 (2001).

[37] N. Kalyanasundaram, M. Moore, J. Freund, and H. Johnson, Acta Materialia 54, 483 (2006).

[38] M. Moseler, P. Gumbsch, C. Casiraghi, A. Ferrari, and J. Robertson, Science 309, 1545 (2005).

[39] . M. Kalff, C. Comsa, and T. Michely, Surf Sci 486, 103 (2001).

[40] R. J. Asaro and W. A. Tiller, Metall. Trans. 3, 1789 (1972).

[41] M. Grinfeld, Sov. Phys. Dokl. 31, 831 (1986). 
[42] C. Madi, H. George, and M.J. Aziz, (unpublished).

[43] For amorphous materials, ion-stimulated viscous flow can be a similarly stabilizing influence $[9]$.

[44] We find our coordinate system more suitable for algebraic manipulations than the one typically used in previous studies, where $\hat{z}$ is taken to be normal to the (unperturbed) surface, and $\hat{x}$ is tangential to it. In the linear dynamics discussed in this paper, the transformation from our coordinate system to this one is obtained by replacing $\partial_{x} \rightarrow \sqrt{1+b^{2}} \partial_{x}$ and $\partial h / \partial t \rightarrow$ $\sqrt{1+b^{2}} \partial h / \partial t$.

[45] Notice that the coefficients $\left\{A_{j}\right\}$ are not unique because the functions $e^{-g_{j}(r)-f_{j}(z)}$ do not form a basis. 